\title{
Pour la démarche géolinguistique de la standardisation des variétés amazighes du Rif
}

\author{
Mena Lafkioui \\ Département des langues et cultures africaines, Université de Gand
}

\begin{abstract}
This article presents a proposition to standardise the Amazigh (Berber) varieties of the Rif (Northern Morocco) on the basis of my geolinguistic study Atlas linguistique des variétés berbères du Rif which provides the data necessary to construct a representative linguistic koinè for the Rif region through computational dialectometry analyses. Dialectometry is a quantitative method to calculate distances between linguistic varieties and to group the latter following different parameters.

Key Words: Tarifit (Amazigh language of the Rif; North Morocco), Geolinguistics, Dialectology, ComputationalDialectometry, Language Planning
\end{abstract}

\section{Introduction}

Le problème le plus ardu auquel les aménageurs (linguistes ou autres) étaient (et sont encore) confrontés est celui de la variation. Par cela, l'on entend généralement la variation dialectale, c'est-à-dire l'ensemble des réalisations phoniques, morphologiques, syntaxiques et lexicales variant en fonction de la localisation géographique. La dispersion géographique des variétés linguistiques arrive à entraver sérieusement l'intercompréhension entre les locuteurs amazighophones du Rif. ${ }^{.}$

Deux autres types de variation caractérisent le domaine rifain et entrent donc en jeu dès qu'on souhaite codifier sa graphie et sa grammaire : la variation sociale et la variation individuelle. La variation sociale couvre plusieurs réalités dont principalement celle de l'habitat (ville/campagne, pays d'origine/pays d'immigration), du sexe, de l'âge, de la classe sociale et du niveau/type d'instruction. Ainsi, l'on peut clairement constater que la demande sociale d'un passage à l'écrit des variétés rifaines vient en ce moment surtout des jeunes étudiants de l'ensemble du Rif. En immigration, la situation est bien différente. Outre certains étudiants de la première ou deuxième génération, ce sont surtout les ar-

Le Rif connaît plusieurs sous-ensembles linguistiques qui se divisent en nombreuses variétés présentant chacune des spécificités sur tous les plans linguistiques. A ce propos, voir Atlas linguistique des variétés berbères du Rif de Lafkioui (2007a). 
tistes (poètes, comédiens et écrivains) s'exprimant auparavant uniquement à l'oral, qui s'intéressent à la codification de leur langue.

La variation individuelle recoupe en partie la variation sociale, mais intègre l'aspect idéo-historique et psychologique de l'individu. Par exemple, le choix du tifinagh (alphabet libyco-berbère) comme support graphique peut chez un militant amazigh être motivé par un rejet idéologique de l'arabe comme langue de l'oppresseur et du latin - symbolisant le français et l'espagnol - comme langue de l'ancien colonisateur. Le tifinagh apparaît alors comme le seul alphabet permettant de restaurer et de promouvoir la langue amazighe dans son intégralité.

Bien que l'IRCAM (Institut Royal de la Culture Amazighe) ait opté officiellement pour les caractères tifinaghs, la préférence des Rifains va toujours encore au latin. Dans cette situation de polygraphie complexe - et fastidieuse pour certains -, le scripteur rifain essaie de trouver une notation qui lui convient le mieux (pour toutes sortes de raisons: psychologiques, sociales, politiques, pragmatiques...), quitte à puiser dans plusieurs systèmes à la fois. Un Rifain résidant au Maroc s'inspire généralement des graphies élaborées par les Imazighen sur place ou par leurs confrères kabyles. Elles sous-tendent des configurations phoniques qui se fondent, à des degrés divers, sur le code phonographique espagnol ou français. Il en est de même pour la pratique scripturale en immigration, avec comme différence que les Rifains y préfèrent parfois employer des systèmes confectionnés en Europe. Naturellement, le néerlandais et l'allemand, langues nationales de pays d'immigration rifaine forte, s'ajoutent à la liste des codes phonographiques élémentaires auxquels ils se réfèrent. Néanmoins, on constate au Maroc et dans les diasporas une volonté explicite d'uniformisation de l'écriture rifaine à base latine. Mais les Rifains n'arrivent pas encore à décider s'il faut réduire la variation rifaine à une seule orthographe pan-amazighe ou essayer d'établir une koinè rifaine qui tient compte de ses spécificités. Nombreux sont ceux qui souhaitent paradoxalement l'intégration complète du tarifit (ensemble de variétés amazighes du Rif) dans le système pan-amazigh, ainsi que la conservation de ses particularités linguistiques. La pratique réelle montre toutefois que la plupart ne parviennent pas à écrire en tarifit sans marquer les propriétés phonétiques de leur variété. ${ }^{2}$

\section{Quelles options pour la construction du tarifit « standard »?}

Les diverses méthodes aptes à la standardisation des variétés amazighes du Rif relèvent de:

- La démarche géolinguistique qui implique la formation d'une koinè représentative du Rif fondée sur la recherche dialinguistique interne;

- La démarche géopolitique qui tient le statut socio-économique comme critère de sélection principal de la variété représentant toute l'aire du Rif. A cet égard, le choix est vite fait : la ville de Nador est le plus grand centre socio-économique du Rif; sa koinè particulière représenterait par conséquent toutes les variétés ama- 
zighes du Rif. Le second centre socio-économique considérable est la ville d'AlHoceima qui est à la fois aussi la référence idéo-historique des Imazighen du Rif occidental et de plusieurs tribus du Rif central. ${ }^{3}$

\section{Pour la démarche géolinguistique de la standardisation des variétés amazighes du Rif}

Au vu de la variation linguistique - particulièrement marquée - du tarifit ${ }^{4}$, l'élaboration d'une koinè de référence se voulant représentative et exhaustive est subordonnée à une étude dialinguistique interne. Celle-ci implique, de par sa nature, la réalisation d'un atlas linguistique, c'est-à-dire un ensemble structuré de cartes géolinguistiques référant à des phénomènes linguistiques précis sélectionnés sur la base de leur degré d'aptitude à l'analyse comparative (Lafkioui, 2007a).

Pour ce faire, des enquêtes de terrain effectuées de façon systématique et portant sur l'ensemble des variétés potentiellement divergentes, étaient indispensables. Elles devaient être fondées sur des enquêtes de types:

- dirigé, faisant appel à des questionnaires dressés en fonction de la nature linguistique de ces variétés. Celles-ci ont montré la nécessité d'inclure à pied égal tous les plans linguistiques intervenant dans la construction d'une étude géolinguistique comparative: la phonétique, la phonologie, la morphologie, la syntaxe et le lexique. Ce choix théorique et méthodologique est particulier dans la mesure où il n'a jamais été pris auparavant dans les études de géographie linguistique amazighe généralement limitées au domaine du lexique. Au fur et à mesure que l'on a progressé dans la collecte et l'exploitation des matériaux, des adaptations et des affinements de ces questionnaires se sont imposées. Car, dans une région très peu explorée comme le Rif (surtout le Rif occidental et méridional), des situations inattendues - linguistiques, mais aussi géographiques et démographiques ont sensiblement altéré le contenu et la forme du questionnaire (Pop, I951: 1075). Il va sans dire que le passage par une langue seconde (arabe dialectal, français ou espagnol) a présenté ici une utilité considérable, dans la mesure où il a permis de minimaliser l'influence (linguistique et autre) possible de l'enquêteur sur l'enquêté.

- semi-dirigé, réalisées par le biais d'interviews dans lesquelles des questions ouvertes traitant des thèmes divers ont été posées. Elles ont été parfois soutenues par des images.

- non-dirigé, où la primauté a été donnée aux expressions verbales et non verbales spontanées, tout en tenant compte de leur diversité formelle et fonctionnelle.

3 Pour la répartition géolinguistique des variétés amazighes du Rif, voir Lafkioui (2007a).

4 Voir Lafkioui (2006a, 2006b, 2007a, 2007b, sous presse I, sous presse 2).

5 Pour l'élaboration des questionnaires et les critères de sélection des points d'enquêtes et des informateurs, voir entre autres : Gilliéron (1902-1910), Basset (1929, 1936, 1939, 1942), Pop (1951), Möhlig (1980), Bouqiaux \& al. (1980), Kurath (1972), Kurath \& B. Bloch (1939), Orton (1960, 1962), Frei (1953), et Trudgill (1975, 1994), Chambers \& Trudgill (1980, 1998), Lafkioui (2007a). 
Dans le souci permanent de représentativité et d'exhaustivité, il a été important de prendre en considération, autant que peut se faire, le caractère oral des textes lors de la transcription (et éventuellement la traduction) des matériaux recueillis; c'est-à-dire, toutes les dimensions (verbales, para-verbales et non-verbales) accompagnant leur production effective, même si elles s'avèrent souvent très difficiles à rendre en graphie.

Comme il n'existait pas de carte géoréférenciée exacte des tribus et villages amazighophones $\mathrm{du}^{\mathrm{Rif}}{ }^{b}$ - accessible au public - il a fallu en concevoir une qui permet en outre des traitements automatiques ${ }^{7}$.

Une fois que la carte des tribus et celle des points d'enquête - toutes deux accompagnées de leur indexe systématique - ont été réalisées, a suivi le procédé de " mapping " qui consiste à articuler les faits linguistiques sur les points géographiques choisis. C'est à ce moment-là que le logiciel de " Mapinfo Professional " est devenu indispensable. Le résultat final était un ensemble de cartes géolinguistiques présentant des phénomènes divers de la linguistique amazighe ${ }^{8}$.

Suit alors le procédé de classification dans le lequel l'atlas linguistique constitue une étape primordiale, car il fournit non seulement les données indispensables à toute autre type de comparaison mais aussi leur localisation géographique exacte. Le schéma suivant résume les méthodes de classification les plus usuelles:

- Atlas linguistique $=$ base de toute autre comparaison ${ }^{9}$

- Autres méthodes de comparaison:

Comparaison historique/génétique

$\rightarrow$ Koinè historique

Comparaison typologique

$\rightarrow$ Koinè typologique

Comparaison dialectométrique

$\rightarrow$ Koinè moyenne"

Chacune de ces méthodes a son mérite. Tout dépend du but scientifique visé. Mais c'est celle de la « dialectométrie " qui forme la suite logique à un atlas linguistique. Elle a comme atout principal d'être une méthode quantitative qui permet de calculer les distances linguistiques entre les variétés linguistiques et de regrouper ces dernières selon des critères variables. Parmi les multiples méthodes en dialectométrie ${ }^{10}$, celle par traitement automatique jouit de notre préférence pour les raisons que:

Les seules cartes des tribus rifaines disponibles (non détaillées cependant) sont celle de Renisio qui remonte à 1932 et celle de Ibáñez de 1944 .

7 Pour des cartes géoréférenciées du Rif (cartes des tribus et des villages du Rif) et leur conception, voir Lafkioui (2007).

8 Voir Lafkioui (2007a).

9 Compte tenu de la spécificité des variétés amazighes, et en l'occurrence celle du Rif, nous avons intégré dans l'Atlas linguistique des variétés berbères du Rif (Lafkioui 2007a), là où cela s'est avéré nécessaire, un nombre considérable d'analyses explicatives d'ordre synchronique et diachronique. 
- la distance et la fréquence y sont mesurées de manière automatique;

- la classification y est numérique;

- des analyses statistiques peuvent être effectuées et visualisées automatiquement;

- la cartographie y est assistée par l'ordinateur.

Au plan de l'aménagement linguistique, la dialectométrie peut fournir une koinè moyenne des variétés rifaines fondée sur la classification quantitative de leurs faits linguistiques suivant le critère de ressemblance/différence. Cette koinè devrait cependant être épaulée par des analyses comparatives de type historique et typologique afin de créer une véritable référence représentant le mieux plus possible la variation linguistique du tarifit ainsi que son uniformité. Il va sans dire que toute koinè linguistique n'est viable que lorsque ses dimensions extralinguistiques (historique, anthropologique et socioéconomique) sont prises en compte dans son application réelle, quelle soit à titre institutionnel (éducation et information nationales surtout) ou individuel.

\section{Références}

BASSET, A. (1929). " Etudes de géographie linguistique en Kabylie », Leroux, Paris.

(1936). "Atlas linguistique des parlers berbères, Algérie ", Territoires du nord, fasc. I, Equidés, Alger.
(1939). " Atlas linguistique des parlers berbères, Algérie ", Territoires du nord, fasc. II, Bovins, Alger.
(1942). "Etudes de géographie linguistique dans le Sud marocain ", Hespéris: 3-22.

BOUQIAUX, L. \& al. (1980). "Problèmes de comparatisme et de dialectologie dans les langues africaines ", Paris, Société d'études linguistiques et anthropologiques de France.

CHAMBERS J. K. \& TRUDGILL, P. (I980, I998 réed.). " Dialectology ", Cambridge, Cambridge University Press.

FREI, H. (1953). "Le livre des deux mille phrases", Genève, Librairie Droz.

GILLIERON, J. (1902-1910). "Atlas linguistique de la France ", I 3 vols., Paris, Champion.

IBÁÑEZ E., I944. " Diccionario español-rifeño ». Madrid.

KURATH, H. (1972). "Studies in Area Linguisics ", Bloomington/London, Indiana University Press.

KURATH, H. \& BLOCH, B. (1939). " Handbook of the Linguistic Geography of New England », Brown University Press.

LAFKIOUI, M. (1999). " Propositions pour la notation usuelle à base latine du berbère et application sur le rifain " In: L' enseignement/Apprentissage de l'amazighe. Expériences, problématiques et perspectives. (Actes du colloque de l'Association de l'Université d'été d'Agadir), Agadir: 66-82.

- (2000). "Propositions pour la notation usuelle à base latine du rifain " In: les Comptes rendus du G.L.E.C.S. XXXIII (30 janvier 1997): 189-200.

- (2002). " Le rifain et son orthographe : Entre variation et uniformisation " In: Codification des langues de France. D. Caubet, S. Chaker \& J. Sibille (eds.), Paris, L'Harmattan: 355-366.

- (2006a). "La vocalisation des alvéolaires $/ \mathrm{r} /$ et $/ \mathrm{rr} /$ dans les variétés berbères du Rif ", Etudes berbères III. Le nom, le pronom et autres articles. (Ibriszimow, Vossen \& Stroomer eds), Köln, Köppe Verlag: $175-184$.

- (2006b). « La spirantisation dynamique de la vélaire occlusive simple $/ \mathrm{k} / \mathrm{dans}$ les variétés berbères du Rif ", In: Studi berberi e mediterranei. Miscellanea in onore di L. Serra, IUO, Napoli: 219-228.

— (2007a). "Atlas linguistique des variétés berbères du Rif. " Köln, Rüdiger Köppe Verlag (In: Berber Studies, Volume I6), 29I (format " $\mathrm{A}_{3}$ ", couleur, 356 cartes +47 tableaux).

— (2007b). "Variation géolinguistique et standardisation des variétés amazighes du Rif ", In: La Culture Amazighe et le Développement Humain (Ennaji, ed), Fès, Presse Universitaire, 69-74. 
_ (sous presse I). "Pour la recherche dialinguistique du berbère. Le cas du tarifit ", In: From Oral Discourse Analysis in Berber to Academic Language Skills in Dutch (25/09/2003, A. Elaissati/Universiteit Tilburg-Babylon), Harrossowitz, io p.

_- (sous presse 2). "Les indices personnels verbaux des variétés berbères du Rif ", dans: L' Amazighe dans l'Oriental et le Nord du Maroc: variations et convergences (Bouhjar \& Souifi eds), Rabat, Editions IRCAM, I2 p.

—_ (sous presse 3). "Analyses dialectométriques du lexique berbère ", Etudes Berbères IV. (Ibriszimow, Vossen \& Stroomer eds), Köln, Köppe Verlag, 19 p.

— (sous presse 4). "Etudes de géographie linguistique berbère. Variation géolinguistique et classification dialectométrique ", Bulletin des Séances de l'Académie Royale des Sciences d'Outre-Mer, Bruxelles, Publications d'ARSOM, $22 \mathrm{p}$.

— (sous presse 5). "Dialectométrie berbère: méthodes et analyses ", Comptes rendus du G.L.E.C.S., XXXVI, $20 \mathrm{p}$.

MÖHLIG, W. J. G. (I980). "L'atlas linguistique du Kenya : méthodes et résultats ", L. Bouqiaux et al. (eds.) Problèmes de comparatisme et de dialectologie dans les langues africaines, 57-83.

ORTON, H. (1960). " An English dialect survey : Linguistic Atlas of England ", Orbis, 9: 393-397.

- (1962). "Survey of English dialects: introduction », Leeds, E. J. Arnold.

POP, S. (195I). " La dialectologie. Aperçu historique et méthodes d'enquêtes linguistiques ". Dialectologie non romane, Louvain/Gembloux, Chez L'auteur / J. Duculot.

RENISIO, A. (1932). "Etude sur les dialectes berbères des Beni Iznassen, du Rif et des Senhaja de Srair ». Paris.

TRUDGILL, P. (1975). " Linguistic geography and geographical linguistics " In: C. Board et al. (eds.) Progress in Geography, vol. VII. Edward Arnold.

TRUDGILL, P. (1994). “ Dialects ", London, Routledge 